\title{
Razones sin lenguaje: el caso de los animales no humanos*
}

\author{
Andrés Crelier \\ Universidad Nacional de Mar del Plata /CONICET, Argentina
}

\begin{abstract}
Resumen: La tesis general del trabajo es que los animales no lingüísticos pueden operar en base a razones. Se sostiene ante todo que la conducta individual flexible de algunos animales ofrece evidencia de racionalidad instrumental. Se argumenta luego que en el marco de los problemas prácticos -como el de la fabricación y uso de herramientas por parte de algunos primates- pueden advertirse procesos de deliberación sin lenguaje. Las razones se interpretan como representaciones mentales y la deliberación como un modo de operar sobre series de representaciones asociadas. Se sostiene, finalmente, que la existencia de una infraestructura comunicativa no lingüística -como la que propone Tomasello- permite ya comunicar razones.
\end{abstract}

Palabras clave: razones; lenguaje; animales no humanos; acción; comunicación

Abstract: "Reasons without language: the case of non-human animals". The paper puts forward the thesis that non-human animals are able to operate with reasons. It argues that the flexible individual conduct is evidence of instrumental rationality and that the context of a practical problem -like tool use by chimpanzees- points toward the existence of deliberative thought. Reasons can be seen as mental representations and deliberation as a way to operate with series of representations. Finally, it is suggested that a communicative infrastructure-such as the one elucidated by Tomasello- makes it possible to communicate reasons without language.

Key Words: reasons; language; non-human animals; action; communication

\footnotetext{
* Una primera versión de este trabajo, "Haciendo plausible la idea de dar razones sin lenguaje: alternativas y objeciones", fue discutida en el I Coloquio sobre Pensamiento Animal. Universidad Nacional de Córdoba, Argentina, en marzo de 2014. Agradezco a los evaluadores anónimos de la revista Areté, cuyos comentarios y correcciones me ayudaron a mejorar este trabajo.
} 


\section{Introducción}

La filosofia reciente ha ofrecido explicaciones plausibles acerca del ejercicio de diversas capacidades cognitivas, desde perceptivas hasta conceptuales, en ausencia de lenguaje. Sin embargo, la capacidad de operar con razones parece reservada para los animales humanos, ya sea como bastión del "lingualismo"1 o como candidato para la diferencia antropológica. Según esto, solo un animal capaz de lenguaje estaría en condiciones de actuar a la luz de razones o de deliberar en base a ellas. En este trabajo me opondré a esta última perspectiva buscando hacer plausible la idea de operar con razones en ausencia de lenguaje. Pienso que este problema no es solo conceptual sino que surge en gran medida de la necesidad de tener en cuenta la evidencia creciente en etología cognitiva. En mi argumentación a favor de la tesis general de que es posible operar con razones incluso en ausencia de lenguaje, pondré el foco en las siguientes capacidades: actuar en base a razones (sección 1), deliberar (sección 2) y comunicar razones sin lenguaje (sección 3). En cada caso procuraré destacar los problemas filosóficos y la evidencia relevante que encontramos respecto a ellos.

En cuanto a la relevancia de este tema, se trata de entender la diferencia antropológica, es decir, no tomarla en un sentido absoluto ni rechazar su existencia. Esto conduce, entre otras cosas, a consideraciones morales. Si es correcto afirmar, como sostienen algunas corrientes filosóficas, que la capacidad de dar razones está ligada con, o es condición de, ser un agente moral -tema que no trataré-, entonces los argumentos a favor de que es posible dar razones sin lenguaje ampliarán en algún sentido la esfera de los seres morales. Sin embargo, el interés a priori por atribuir derechos a los animales no debe empañar ni sesgar la discusión filosófica. De hecho, ampliar la esfera moral puede ir de la mano con reforzar la consideración moral del propio animal humano, reforzando con ello también su diferencia específica en este aspecto. En el mismo sentido, una mirada naturalista sobre el modo en que los animales humanos y no humanos son capaces de operar con razones puede echar luz sobre el modo específicamente humano de ser moral.

\footnotetext{
1 Esta expresión es utilizada por H. Glock para referirse a la posición según la cual el lenguaje es condición de otras capacidades cognitivas ( $c f$. Glock, H., La mente de los animales. Problemas conceptuales, Oviedo: KRK Ediciones, 2009.)

ARETÉ Revista de Filosofía, vol. XXVIII, N² 2, 2016 / ISSN 1016-913X
} 
1. Actuar en base a razones: el perfil de la racionalidad instrumental y la acción individual en base a razones

Mi tesis en este apartado es que podemos explicar la conducta individual de determinados animales sin lenguaje en base a la estructura de la racionalidad instrumental y como un caso de actuar en base a razones. Tanto en esta sección como en el artículo en general, el foco estará puesto en la acción instrumental en la que el agente mismo comprende las razones por las que actúa, o dicho de otro modo, en la cual las razones lo son para el individuo (en oposición a la especie). Sin embargo, en esta sección haré algunas consideraciones sobre las conductas que, si bien se explican en base a dicha estructura, no conllevan dicha comprensión por parte del individuo. Lo haré justamente para resaltar por contraste la racionalidad instrumental "auténtica" y para reflexionar sobre la transición entre ambas.

La racionalidad instrumental opera sobre una estructura de medios y fines. Las explicaciones que ofrecemos en base a esta estructura poseen aspectos descriptivos y normativos. Así, explicamos una acción en base a los fines perseguidos, los cuales establecen las normas frente a las cuales juzgamos si los medios para alcanzarlos son buenos, adecuados, errados, etcétera. A su vez, una acción se puede explicar en base a una comparación entre medios: suponiendo un fin determinado, la acción que sirve como medio se comprende en parte en contraste con otros medios posibles. Desde un punto de vista evolutivo, la acción de muchos animales no humanos puede describirse en base a este esquema de racionalidad instrumental, lo cual no siempre implica atribuir racionalidad al respectivo agente individual. En caso de animales sin lenguaje, esta atribución se basa principalmente en la conducta observable. Solo una conducta "flexible" puede ser indicio de una acción individual en base a razones. Esta flexibilidad de la conducta indica la existencia de procesos de razonamiento instrumental que no se reducen a razonamientos por asociación fruto de condicionamientos (clásicos o instrumentales). En estos últimos, los estímulos establecen asociaciones que podemos observar en las conductas, las cuales pueden incluso ser relativamente complejas. Los argumentos que rechazan explicaciones exclusivamente basadas en mecanismos asociativos pueden verse entonces como un paso inicial para justificar la atribución de acción individual en base a razones. Para ilustrarlo, traigo brevemente a colación el conocido experimento con ratas de 
Dickinson y Balleine ${ }^{2}$. El experimento muestra conductas que parecen guiadas por un proceso de razonamiento que no puede entenderse como exclusivamente asociativo producto de condicionamientos. Dicho simplificadamente: las ratas no son entrenadas para obtener bebida en oposición a alimento, pero logran actuar como si hubieran comprendido esta distinción al margen del proceso de condicionamiento y como si fueran capaces de utilizarla en un razonamiento instrumental que conduce a una conducta nueva, que puede así considerarse no condicionada.

Se abre aquí una discusión acerca de cómo interpretar los experimentos mencionados, sobre cuáles son los criterios más adecuados para elaborar las explicaciones de las respectivas conductas y rechazar explicaciones de nivel más "bajo", etcétera. Sin entrar en estas cuestiones, me interesa aludir a cierto consenso interpretativo que apunta hacia el escepticismo respecto de que toda conducta animal sea completamente explicable mediante la postulación de procesos asociativos fruto de condicionamientos.

Como descripción filosófica, puede postularse la existencia de una mente que opera con razones sin estar determinada por el entorno inmediato, tomando distancia del mismo y deliberando sobre fines y medios de acción. La tradición filosófica toma estas notas como características de tener "mundo" (aunque se suele asumir que es el lenguaje el que permite satisfacer las condiciones anteriores) ${ }^{3}$.

Se abre aquí un campo de transición muy complejo. Proponer condiciones minimas para operar con razones no implica que, cumplidas estas condiciones, estemos ya en presencia de conductas explicables exclusivamente en base a razonamientos instrumentales o auténticas deliberaciones. Así, entre el condicionamiento -y los procesos de asociación a los que da lugar- y una auténtica deliberación pueden existir capacidades cognitivas que guíen la acción de otra manera, por ejemplo mecanismos que den por resultado las mismas conductas que los mecanismos deliberativos. La conducta legendaria del perro de Crisipo es aquí un buen ejemplo, dado que los experimentos parecen indicar que los perros no razonan inferencialmente en situaciones semejantes, aunque sea natural reconstruir su conducta como

\footnotetext{
2 Dickinson, A. y B.W. Balleine, "Causal Cognition and Goal-Directed Action", en: Heyes, C. y L. Huber (eds.) The Evolution of Cognition, Cambridge Mass.: Cambridge University Press, 2000.

${ }^{3}$ Cf. McDowell, J., Mind and World, Cambridge Mass.: Harvard University Press, 1996; y Crelier, A., "Distancia y mundo. La diferencia específica de los animales humanos sobre el trasfondo de la tradición filosófica", en: Nuevas Fronteras de Filosofia Práctica, v. I (2013), pp. 84-105. Disponible en: http://nuevasfronterasdefilosofiapractica.com/2013/09/23/numero-1-agosto-de-2013/
} 
si lo hicieran ${ }^{4}$. Otro ejemplo de conducta de carácter adaptativo que puede interpretarse mediante el modelo de racionalidad instrumental es el de los castores que construyen diques. Aquí la conducta es, desde cierto punto de vista, flexible (lo cual incita a entenderla como individual), pero desde otro punto de vista es condicionada (lo cual pone de relieve que se trata de una adaptación de la especie).

Esto ubica a los casos de transición en una suerte de paradoja, pues diferentes casos de lo que aparenta ser un mismo tipo de conducta instrumental pueden ser el producto de mecanismos muy diferentes, como si la conducta meramente adaptativa fuera el modelo para la conducta individual guiada por razones. En el terreno de la especulación: ¿puede afirmarse que la especie o la "naturaleza" tiene razones para actuar? Afirmarlo equivale a personificar infundadamente mecanismos naturales, pero negarlo implica asumir que las mismas conductas pueden obedecer a mecanismos de naturaleza muy diferente. En este último caso, ¿cómo puede ser que los mecanismos todavía asociativos que permiten de algún modo una transición hacia la acción auténtica en base a razones sean de una naturaleza diferente a los que operan en la racionalidad instrumental auténtica? Se trata del paso del hambre como causa al hambre como razón.

En lugar de mantener la contraposición, puede pensarse que los razonamientos que utilizan mecanismos asociativos y los que operan genuinamente en base a razones no necesariamente se oponen. Es posible pensar que la operación en base a razones puede "encarnarse" o valerse (completa o parcialmente) de mecanismos asociativos más o menos complejos pero todavía rígidos o mecánicos, o, asimismo, que la operación en base a razones puede apoyarse en mecanismos asociativos volviéndolos más flexibles. Esto permite pensar en niveles y modos de racionalidad práctica, especialmente en sus momentos incipientes ${ }^{5}$. Más allá de este espacio de transición, mi objetivo principal en esta sección ha sido justificar la tesis de que la acción animal individual puede entenderse como acción basada en razones. La justificación se apoyó en el contraste de la acción instrumental "auténtica" frente a conductas explicables en base a modelos más "bajos", como los que recurren a asociaciones (en principio, entre estímulos). Ahora bien, la acción individual basada en razones supone la capacidad de ponderar razones, es decir, de deliberar. Teniendo en cuenta

\footnotetext{
4 Cf. Andrews, K., Do Apes Read Minds. Toward a New Folk Psychology, Cambridge Mass./ Londres: The MIT Press, 2012.

5 Cf. Bermúdez, J.L., Thinking without Words, Oxford: Oxford University Press, 2003, capítulo 6. 
eso, en el próximo apartado me detendré en el contexto práctico en el que es posible advertir procesos de deliberación y en algunos problemas filosóficos que derivan de alli.

\section{Deliberar en base a razones}

\subsection{El problema práctico y el caso del uso de herramientas}

En esta sección sostendré que algunos animales llevan a cabo procesos de deliberación sin lenguaje en el marco de un problema práctico. Visto de otro modo, sostendré que estos procesos de deliberación pueden inferirse a partir de situaciones problemáticas como la fabricación y uso de herramientas por parte de algunos primates.

Volvamos a la acción individual basada en razones. Un caso auténtico debe cumplir al menos dos condiciones: las razones deben serlo para el agente y este debe adoptarlas "libremente". En el caso de la racionalidad instrumental que he descrito, se trata de dos requisitos ligados entre si, pues para que las razones lo sean para el agente, este debe haberlas adoptado libremente. Esto último se advierte cuando es legitimo suponer que el agente ha optado dejando de lado posibilidades de acción no realizadas. La libertad en un sentido absoluto no solo es un concepto difícil de definir, sino que tampoco es comprobable en la conducta, pero sí lo es la libertad en un sentido relativo, medido con respecto a acciones realizables pero no realizadas. Los dos requisitos mencionados equivalen a afirmar que el contexto de posibilidades de acción deben serlo para el agente mismo.

Un contexto en el que se advierte esta clase de racionalidad es el problema práctico, donde el hecho de tener una meta está ligado con la comprensión de las dificultades para alcanzarla. Se trata de una sensibilidad a que las circunstancias no sean las adecuadas para conseguir la meta. Esto genera un marco en el que el agente tiene un fin y actúa eligiendo -con un margen de libertadentre una variedad de posibilidades de acción (en principio, los medios para alcanzar el fin). Para que podamos hablar de un problema, debe haber entonces posibilidades de acción diferentes para solucionarlo (la solución equivale a la meta). Y si bien la flexibilidad en la adopción de los fines no es una condición igualmente necesaria, creo que los animales que resuelven problemas tienen también a su disposición un mínimo conjunto de metas entre las cuales elegir, aunque se trate de pequeñas variaciones entre fines relativamente impuestos por condicionamientos biológicos. (Más aun, puede pensarse que ser capaz de 
elegir entre medios diferentes para alcanzar un mismo fin fomenta la capacidad de elegir entre metas diferentes. Entender que algo puede ser un medio adecuado para obtener un determinado fin supone que también podría ser un medio inadecuado, y de aquí hay una distancia muy corta a entender que podría ser un buen medio para obtener una meta diferente, lo cual supone una cierta flexibilidad en cuanto a la adopción de metas).

Finalmente, para incluirlos en el repertorio a disposición del agente, estas metas y medios deben ser realizables, y para asegurarnos de ello, debe constatarse que han sido realizadas alguna vez, al menos por otros miembros de la misma especie. El hecho de que o bien el agente o bien otro miembro de su misma especie haya tomado decisiones diferentes (a menudo sin éxito), es un indicio de distancia del entorno y le da sentido a la posibilidad de errar (en la pretensión de cumplir una meta o realizar un medio para alcanzarla), permitiendo suponer un cierto grado de libertad, pues no habrá de suponerse una relación univoca entre estímulos y respuestas conductuales. Hemos llegado al siguiente punto: el marco de un problema práctico permite entender la capacidad de deliberación. Un ejemplo que ilustra esto es la fabricación y uso de herramientas para cazar termitas por parte de chimpancés, capacidad que se encuentra documentada ${ }^{6}$. Aquí podemos suponer la posibilidad de adopción de diferentes metas y diferentes medios para alcanzarlos. La posibilidad de elegir entre metas diferentes puede suponerse en base a las diferentes y complejas líneas de acción que adoptan los chimpancés estudiados, definidas por los fines a alcanzar: cazar termitas y hormigas de diferentes especies, extraer miel de panales, limpiarse con hojas, etcétera. Ciertamente, estas lineas de acción son en parte motivadas por el entorno y la herencia, pero también son adoptadas de modo flexible: los chimpancés que son observados por humanos no persiguen los mismos fines que aquellos que no son observados, las hembras son más proclives a fabricar herramientas para cazar termitas que los machos, grupos de chimpancés que viven en distintas regiones de África han desarrollado diferentes variantes conductuales, etcétera.

En particular, la adopción de los medios revela el propósito de adecuarlos a cada fin: el largo y el tamaño de la rama, su limpieza y preparación, etcétera, dependen de la línea de acción que los chimpancés han encarado. Puede afirmarse que cada meta posee sus medios más adecuados y que es implícitamente

\footnotetext{
6 Boesch, C. y H. Boesch, "Tool Use and Tool Making in Wild Chimpanzees", en: Folia Primatologica, v. LIV (1990), pp. 86-99. 
acompañada por una variedad de medios posibles que resultarian inadecuados, algunos de ellos porque pertenecen a otras líneas de acción instrumental. Finalmente, una evidencia adicional de conducta instrumental es el hecho de que en ocasiones los agentes modifican las herramientas luego de sus primeros intentos de uso.

Esta variedad de conductas a disposición, que no son las mismas para distintos grupos de chimpancés, indican un grado de distancia del entorno y libertad individual. Podemos describir así la situación: en algunos grupos un chimpancé hembra adulto se encuentra frente a una situación que vive como un problema práctico, delibera sobre el mejor modo de resolverlo y persigue finalmente líneas de acción tendientes a alcanzar la meta propuesta.

Los criterios para interpretar la conducta no solucionan diversos problemas filosóficos referidos a la atribución de pensamiento sin lenguaje, por ejemplo la legitimidad de atribuir a los animales capacidades conceptuales y doxásticas. No es este el lugar de abordarlos, dado que mi interés se dirige específicamente hacia la acción guiada por razones. Me enfocaré, en las subsecciones que siguen, en algunos problemas que afloran cuando intentamos esclarecer cómo son los procesos de razonamiento instrumental que de hecho he atribuido al interpretar conductas como las de los chimpancés: ¿son las razones hechos o representaciones?, ¿cómo entender la auto-cognición en la deliberación?, ¿son los procesos deliberativos de carácter inferencial?

\subsection{Las razones como hechos o como representaciones}

Entre los problemas filosóficos que surgen a la hora de entender las razones está el de si se trata de hechos del mundo o de estados mentales. Esta opción resulta relevante en nuestro contexto porque en cada caso conduce a otros problemas o los evita. Por un lado, si las razones son estados mentales, surge el problema de si esto requiere la capacidad de dirigir la mente hacia sí misma (auto-consciencia o meta-cognición), o al menos de operar expresamente con los propios estados mentales, lo cual estaría fuera del alcance de las mentes a-lingüísticas. Por otro lado, si se considera que las razones son directamente hechos del mundo, surge el problema de cómo entender la relación intencional entre la mente y una variedad de géneros o sub-géneros de lo que usualmente consideramos razones y que a menudo no parecen ser hechos del mundo. Detengámonos en esta última opción de las razones para actuar como hechos del mundo, elegida por ejemplo por Glock, quien pretende evitar en parte los problemas de la auto-consciencia en la acción racional y algunos reparos 
"lingualistas" asociados7. Glock sostiene que las razones lo son para el agente, pero que hay que entenderlas como hechos del mundo. A mi modo de ver, esta opción conlleva dos riesgos: 1) como él mismo advierte, conduce a la dificultad de explicar razones que no son hechos verdaderos; 2) puede inducir a considerar las razones como entidades fuera de la relación intencional.

Sobre lo primero, si las razones son hechos, hay que explicar la relación intencional existente no solo entre la mente y las razones que son hechos verdaderos del mundo, sino entre la mente y otros tipos de razones que no son claramente hechos verdaderos. Se forzaría en este último caso la propia noción de "hecho", al menos su sentido corriente, pues habria que hablar de hechos posibles, hechos negativos, alucinaciones qua hechos, etcétera. Guiados por la forma gramatical común de dar razones, hay que suponer que la relación intencional es del mismo tipo en todos los casos -lo cual se presta a contraejemplos- o abrir el espacio para explicaciones diferentes para tipos de razones diferentes, lo cual despierta la sospecha de que se elaboran explicaciones ad hoc.

Por el contrario, si las razones -incluso si son también hechos-, son también representaciones de hechos, la cuestión se simplifica, pues el agente guarda en todos los casos relación con sus propias representaciones, término que puede englobar además de los hechos verdaderos, hechos posibles, representaciones alucinatorias y negativas de hechos, entre otras posibilidades. Tendriamos una sola clase de relación intencional para casos como los siguientes:

a) Tomó el paraguas porque llueve.

b) Dejó el paraguas porque no llueve.

c) Dejó el paraguas porque creía que no llovía.

d) Tomó el paraguas porque vio llover (a raíz de haber ingerido una sustancia alucinógena).

El segundo riesgo mencionado de ver a las razones como hechos es perder de vista la relación intencional. Justamente, creo que conviene enfatizar que las razones no son nada fuera de dicha relación. Las razones solo tienen carácter de razones para alguien que las considera como tales. Pero el modo lingüístico de expresar las razones suele hacer perder de vista esta relación. Glock indica con razón que en el modo de expresar razones puede y suele no aparecer el verbo intencional "creer". En efecto, no digo "tomo el paraguas porque creo que llueve" sino "tomo el paraguas porque llueve". Pero incluso

\footnotetext{
7 Tendré especialmente en cuenta en lo que sigue a Glock, H., "Can Animals Act for Reasons?", en: Inquiry, v. LII, 3 (2009), pp. 232-254.
} 
esta última formulación expresa una relación intencional que tiene dos polos. El modo de expresar lingüísticamente las razones sin verbos que, como "creer", hacen patente la relación intencional, y la noción correspondiente de que son hechos, puede hacer perder de vista este rasgo de lo mental.

En este punto uno podría preguntarse lo siguiente: si la creencia está implicada en toda acción en base a razones, ¿por qué a menudo no aparece el verbo creer en la formulación lingüística que la expresa? Una razón de su ausencia podría ser su carácter omnipresente, cuya articulación sería superflua, pero creo que la explicación es otra. El verbo "creer" tiene dos aspectos semánticos: uno "pro-cognitivo", que sugiere conocimiento, y otro "anti-cognitivo", que indica duda y perspectiva subjetiva. De este modo, cuando una razón nos motiva al punto de que actuamos en base a ella no tiene sentido articular (o correr el riesgo de aludir a) una duda sobre ella ni sugerir que se trata de una mera perspectiva del mundo, y por ello no resulta adecuado introducir un verbo con la ambigüedad mencionada. A lo sumo lo haremos si somos interrogados sobre las razones de nuestra acción, por ejemplo en un juicio. Pero fuera de contextos como el del juicio, no solemos -con la correcta motivación de no resultar contradictorios- usar este verbo, al menos en primera persona. Dicho brevemente, cuando creemos con intensidad (lo cual es el caso paradigmático cuando una razón nos motiva) el verbo "creer" tiende a desaparecer de la formulación lingüística de las razones, pero esto no implica que desaparezca la relación intencional con una representación creída.

Si esto es así, entonces una razón sin lenguaje puede ser una representación de un hecho en el que creemos y que motiva a su vez a una acción. Así, pienso que las razones son hechos representados por el agente, al margen de que lo representado sean también hechos del mundo cuando las representaciones son verdaderas. Al tratarse de una relación intencional, no puede legítimamente hablarse de razones fuera esta relación, así como tampoco tiene sentido concebir una actitud práctica deliberativa que no tenga como objeto intencional un conjunto posible de razones.

\subsection{Auto-cognición y razones}

Al evitar los problemas de tomar a las razones como hechos, y al admitir que son representaciones de hechos, regresan los problemas ligados con la meta-cognición y la estructura del pensamiento que es tomado como objeto. En el caso humano lingüístico, podemos suponer la capacidad de auto-cognición entre otras cosas porque la podemos articular lingüísticamente y porque el 
lenguaje ofrece una estructura adecuada para ser objeto del pensamiento. En el caso de animales no lingüísticos se abre en cambio un abanico de problemas.

¿Qué tipo y grado de meta-cognición exige la relación intencional con las razones sin lenguaje? No es suficiente (aunque sí un requerimiento básico) poseer una auto-cognición mínima, por ejemplo sentir el propio cuerpo y ser capaz de ubicarse en el espacio. Hace falta también poseer una capacidad de operar con contenidos mentales propios, lo cual supone distancia del entorno y libertad de elección. A mi modo de ver, se trata de una cuestión empírica que puede esclarecerse en gran medida observando e interpretando la conducta no verbal. Si la única o la mejor explicación de la fabricación de herramientas por parte de chimpancés requiere postular una suerte de auto-cognición, se tratará de una atribución justificada.

Sin embargo, existen otros problemas que relacionan la auto-cognición y la capacidad de operar con razones (entendidas como representaciones). Bermúdez introduce aquí una tesis contra la auto-cognición sin lenguaje: el ascenso intencional (pensar sobre los propios pensamientos) requiere un ascenso semántico, y esto último solo es posible si el pensamiento está vehiculizado lingüísticamente ${ }^{8}$. Se trata, asimismo, de una concepción inferencialista de la meta-cognición: "Second order cognitive dynamics involves sensitivity to the inferential relations between thoughts" . Para oponernos al requerimiento del lenguaje, se pueden sostener dos cosas. Primero, la necesidad del lenguaje puede cuestionarse en base a modelos conexionistas, probabilistas o cartográficos que soporten inferencias ${ }^{10}$. Segundo, puede ponerse en cuestión la propia concepción inferencial del pensamiento involucrado en la meta-cognición.

Es esta segunda opción la que voy a seguir. Se trata de postular que no toda cognición de segundo orden requiere sensibilidad a las relaciones inferenciales (aunque en algunos casos sea reconstruible como una inferencia). Estos son algunos contraejemplos de dinámica cognitiva de segundo orden no inferencial:

- La auto-consciencia pura: para los filósofos modernos de la conciencia toda actividad de pensar es necesariamente auto-consciente, tratándose de algo más básico que la sensibilidad inferencial. Para Kant el "yo pienso" debe poder acompañar todas mis representaciones, al margen de que ese acompañamiento requiera en principio capacidad inferencial o lenguaje.

\footnotetext{
8 Bermúdez, J.L., Thinking without Words, pp. 158ss.

9 Ibid., p. 161.

10 Camp, E., “Thinking with Maps”, en: Philosophical Perspectives, v. XXI, 1 (2007), pp. 145-182.
} 
- La actividad de recordar: se trata de tener presente algo que sé que me pertenece, sin sensibilidad particular a las relaciones inferenciales aunque sí a las relaciones temporales y espaciales entre los recuerdos.

- La realización de una destreza: hay auto-cognición (del lugar que ocupo, de mis movimientos, que auto-corrijo, etcétera) sin que esto involucre una capacidad inferencial (lo cual se advierte en la dificultad para expresar mediante el lenguaje el grano fino de las destrezas y las relaciones entre las mismas).

Estos ejemplos muestran que existen procesos cognitivos complejos de segundo orden de carácter no inferencial. Sin embargo, es posible insistir en que operar con razones da lugar a procesos cognitivos que sí son inferenciales. Se requiere entonces alguna noción de pensamiento deliberativo no inferencial, y mi intuición es que se la puede hallar en la capacidad para operar sobre representaciones asociadas.

\subsection{Hacer inferencias $u$ asociar representaciones}

Podemos describir las acciones realizadas en base a razones en la forma de un razonamiento práctico, pero esto no significa que la reconstrucción lingüística sea de la misma naturaleza que el proceso mental mismo, ni siquiera en el caso humano. Como ha afirmado Norman Malcolm, la forma gramatical no es un indicio de realidad psicológica ${ }^{11}$. Si por ejemplo-glosando de manera libre un ejemplo de Malcolm- alguien advierte que Juan está caminando con mucha precaución y le pregunta a otro observador por qué lo hace, la respuesta puede ser: porque se ha dado cuenta de que el suelo está resbaloso. No obstante, esta respuesta, que reconstruye una razón, no implica suponer que la proposición "el suelo está resbaloso" -que expresa además su razón para actuar- estuvo presente en la mente de Juan ${ }^{12}$. Y exactamente lo mismo sucede con la atribución de pensamiento a animales sin lenguaje.

Frente a esto, Davidson ha argüido que de todos modos debe ser posible para el agente realizar la respectiva reconstrucción inferencial y lingüística del razonamiento práctico ${ }^{13}$. Es decir, si hacemos la atribución suponemos que el agente tuvo y tiene la capacidad de hacer esta reconstrucción, para lo cual se precisa finalmente un lenguaje que pueda expresar actitudes proposicionales, lo cual excluye a los animales sin lenguaje de la acción intencional y racional

\footnotetext{
${ }^{11}$ Malcolm, N., "Thoughtless Brutes", en: Proceedings and Addresses of the American Philosophical Association, v. XLVI (1972-1973), p. 14.

12 Ibid., p. 16.

13 Davidson, D., “Tener la intención”, en: Ensayos sobre acciones y sucesos, Barcelona: Crítica, 1995.
}

ARETÉ Revista de Filosofía, vol. XXVIII, N² 2, 2016 / ISSN 1016-913X 
en general (justamente, para Davidson, la acción se explica de manera central en base a creencias, que son estados intencionales).

Esta concepción se expone a contraejemplos como el siguiente: si nuestros antepasados hominidos se hubieran extinguido justo antes de desarrollar el lenguaje, ninguna de sus conductas técnicas explicables en base a razones, como la fabricación de herramientas, habrian sido acciones auténticas (y menos aun instrumentales), lo cual resulta contra-intuitivo. Para mantener en pie la tesis davidsoniana frente a tales contraejemplos (que podrian ampliarse abarcando homínidos no antepasados nuestros, primates contemporáneos y niños pre-lingüísticos) habría que asumir una cierta posición idealista, sosteniendo no solo que ha de ser posible reconstruir lingüísticamente el sentido de las acciones de esos agentes, sino que tanto su sentido como su carácter de acción es otorgado por nuestras interpretaciones lingüísticas posteriores, dependientes de un desarrollo posterior evolutivamente contingente. Esto nos invita a abandonar el idealismo y pensar que el sentido de la acción instrumental no es ofrecido por inferencias lingüísticamente formulables. A su vez, nos permite pensar que los procesos mismos que guian las acciones en base a razones no son en sí mismos inferenciales según el modelo lingüístico. Se trata, pues, de pensar la deliberación sin lenguaje y de manera no inferencialista.

Una opción que me interesa explorar utiliza en parte explicaciones asociacionistas (aunque no reductivistas). Así, deliberar sobre razones puede entenderse como un proceso que opera sobre representaciones mentales asociadas. Para enfatizar el lado práctico de estas representaciones, pensemos en la noción de Millikan de representación pushmi-pullyu, de carácter descriptivo e imperativo a la vez ${ }^{14}$. Puede pensarse que estas representaciones se encuentran ordenadas en un sentido temporal, como cuando se planifica una acción compleja $^{15}$. Si es expuesto repetidamente a la correspondiente actividad por parte de algunos adultos, un chimpancé joven puede aprender, es decir, formarse representaciones acerca de una serie de acciones conducente a fabricar una herramienta para cazar termitas. Esta serie de representaciones se encuentran intimamente ligadas con la acción. Cuando el agente está motivado por un fin

\footnotetext{
${ }^{14}$ Millikan, R.G., "Pushmi-Pullyu Representations", en: Tomberlin, J. (ed.), Philosophical Perspectives, Atascadero: Ridgeview Publishing, 1996, pp. 185-200.

${ }_{15}$ Papineau comprende justamente al razonamiento instrumental como una adaptación humana independiente del lenguaje. Cf. Papineau, D., "The Evolution of Means-End Reasoning”, en: Royal Institute of Philosophy Supplement, v. XLIX (2001), pp. 145-178.
} 
determinado, al percibir por ejemplo un nido de termitas, puede comprender el problema práctico, evocar dichas representaciones y pasar con ellas a la acción.

Este cuadro no es inferencialista. No requiere operadores lógicos o "protológicos", basta con disponer de series de las representaciones adecuadas. La deliberación se enfocaría en ellas, eligiendo series diferentes o recombinando elementos de series conocidas. Por ejemplo el razonamiento "no hay un león ahora en el estanque, entonces puedo bajar a beber", cuya reconstrucción lingüística incluye una negación, no tiene por qué corresponderse con un proceso real de pensamiento en la mente de una gacela. Basta para ella con la representación de la secuencia adecuada y la sensibilidad a su adecuación.

De todos modos, ¿por qué nos vemos forzados a formular razonamientos prácticos claramente inferenciales cuando reconstruimos conductas como algunas de las mencionadas? Esta necesidad indicaría que, si bien el contenido de las representaciones no posee en sí misma una forma lógica, la capacidad cognitiva que opera sobre ellas sí posee una facultad inferencial o una sensibilidad a las relaciones inferenciales, de modo que estariamos desplazando el problema.

La necesidad de reconstruir inferencialmente sugiere dos alternativas para explicar determinadas conductas de animales sin lenguaje. O bien es preciso atribuir a agentes sin lenguaje alguna suerte de proto-lógica (Bermúdez), -mejor dicho proto-sensibilidad a la lógica-, o bien se puede pensar a la lógica como una abstracción a partir de razonamientos que operan con contenidos sustantivos (desechando aquí los aspectos motores de las representaciones). En este caso, existe sensibilidad a las relaciones entre los contenidos. La reconstrucción lingüística al modo de una inferencia sería un modo de hacer explícitas (Brandom) las relaciones implícitas que existen entre contenidos representativos, pero no posee fuerza normativa sobre los mismos. Se trata de problemas que exceden este trabajo, basta con indicar que esta última opción resultaría más adecuada para el esbozo que he propuesto.

\section{Comunicar razones sin lenguaje}

En la primera sección sostuve que algunas conductas animales no lingüísticas pueden interpretarse como acciones individuales basadas en razones. En la segunda sección argumenté que a partir de determinadas conductas es posible inferir procesos de deliberación que se enfrentan a determinados problemas prácticos. La fabricación y uso de herramientas por parte de chimpancés fue 
la ilustración destacada. En ningún caso se supuso la capacidad de comunicar las razones de la acción, tema del presente apartado.

En principio, es concebible pensar agentes que actúen en base a razones pero que no sean capaces de comunicarlas. Al entrar en el ámbito de la comunicación de las razones, los requerimientos cognitivos son otros, e incluyen la comprensión de la situación comunicativa por parte del agente, que ahora es un interlocutor, y la comprensión del otro como interlocutor comunicativo, entre otros presupuestos. A su vez, esta comprensión debe entenderse de modo recursivo: sé que el otro sabe que yo sé, etcétera.

¿Se pueden cumplir estos requerimientos en algunos animales sin lenguaje? Me inclino por una respuesta positiva. Para ilustrarlo mencionaré el resultado de un experimento de Tomasello y Call con chimpancés criados en un contexto humano, que han aprendido a señalar para pedir alimento. Según estos autores, cuando un chimpancé observa que un humano necesita una herramienta para alcanzarles alimento, y esta herramienta ha sido escondida durante la ausencia del humano, el chimpancé le indica dónde está la herramienta al humano que ha regresado ${ }^{16}$. Tomasello interpreta esto como un gesto imperativo mediante el cual se pide una cosa -la herramienta- como medio para alcanzar otra cosa, el alimento. No es un gesto informativo, pues cuando la herramienta es para uso humano, los chimpancés no realizan este gesto.

Una interpretación posible de esta situación es que el chimpancé comprende una situación problemática y sabe que esta puede resolverse mediante el empleo de racionalidad instrumental. Sobre esa base ofrece al humano una razón para actuar, como si expresara lo siguiente: "le pido a usted la herramienta escondida alli para resolver mi problema de conseguir un alimento escondido". Así, el agente no ofrece razones de la propia acción al modo de una explicación o justificación, sino que comunica imperativamente a un interlocutor razones para actuar, pues supone que este cooperará en la resolución práctica del problema. La herramienta señalada es en ese contexto comunicativo una razón para actuar porque representa la mejor solución, desde el punto de vista del agente, a un problema práctico comprendido por los interlocutores.

Siguiendo con esta lectura, si el humano accede a buscar la herramienta escondida, puede darle sentido a su acción, por ejemplo ante quien lo interroga sobre qué está haciendo en ese momento o por qué lo está haciendo, como sigue: "la razón de mi acción es buscar esta herramienta para resolver el problema

\footnotetext{
${ }^{16}$ Tomasello, M., Origins of Human Communication, Cambridge Mass.: The MIT Press, 2008, p. 35. 
de X -y comprendido por mí- de obtener alimento escondido". El problema práctico forma parte del contexto comunicativo, lo cual permite que las razones puedan ser comunicadas. En este marco, el señalar puede entenderse como la indicación pública de una representación-acción ${ }^{17}$.

Una posible objeción al intento por generalizar esta capacidad es que los primates en cuestión (los referidos por Tomasello) poseen ya un sistema comunicativo que les ha sido enseñado por humanos. Sin embargo, como sostiene convincentemente Tomasello, estos poseen ya una "infraestructura" previa incluso al contacto con humanos. Los elementos de esta infraestructura permiten tanto la comunicación como el posterior desarrollo de una lengua natural como la humana, caracterizada entre otras cosas por su compleja dimensión sintáctica. Sin este último desarrollo, el sistema semiótico que permite dar las razones en la situación descrita por Tomasello no permite, por ejemplo, combinar unidades para formar unidades de un nivel más complejo. De hecho, este autor reseña que ni siquiera los primates que han aprendido gran cantidad de símbolos son capaces de combinarlos mínimamente y en un sentido comunicativamente relevante.

A pesar de ello, la flexibilidad comunicativa del gesto de señalar, que funciona gracias a la comprensión de entornos y problemas compartidos, y gracias también a una comprensión de la situación comunicativa, les permite a los chimpancés comunicar una gran variedad de cosas, casi siempre en modalidad imperativa. Puede así afirmarse que sus gestos cobran sentido en un marco holista no completamente semiótico: es la situación y no (solamente) un conjunto implícito de signos lo que los vuelve comprensibles (en tanto signos). Así, pocas herramientas comunicativas (semióticas) alcanzan para ofrecer una solución, y una solución puede entenderse como una razón (o "proto-razón") para actuar.

Antes de pasar a las conclusiones, me interesa destacar una serie de problemas que se abren en este punto. Respecto de la comprensión de la actividad comunicativa y sus presupuestos: ¿pueden darse razones si no se tiene ninguna noción, aunque sea implícita, de que existen razones mejores que otras, que las razones son por principio comunicables a otros interlocutores, etcétera? Con otras palabras, las razones que se dan en el marco de una

\footnotetext{
${ }^{17}$ El gesto de señalar ofrece también a los humanos lingüísticos la posibilidad de ofrecer razones para actuar sin utilizar el lenguaje. Si ambos sabemos que tú estás apurado por llegar a un determinado sitio, mi dedo indicando un taxi puede interpretarse como una razón para resolver el problema práctico relevante.
} 
infraestructura semióticamente pobre como la que describe Tomasello, ¿poseen los mismos presupuestos comunicativos que los implicados en el uso de una lengua natural?

Otra cuestión es la siguiente: la existencia de un problema compartido por dos o más interlocutores comunicativos, ¿abre la posibilidad de ofrecer una variedad indefinida de razones correspondientes a los aspectos relevantes de lo compartido? El chimpancé que señala comunicativamente un medio para resolver un problema, ¿tiene entonces la capacidad de comunicar otras razones sobre las que también delibera, por ejemplo al planificar la construcción de una herramienta para cazar termitas? Quizás esto no sea posible, tanto por las pocas motivaciones comunicativas y la orientación centrada en el propio ego, como por el poco desarrollo correspondiente de recursos semióticos. De modo que las razones comunicables serian un pequeño subconjunto de las razones a la luz de las cuales muchos agentes actúan.

Las razones que tomo en cuenta son las que tienen una fuerte relación con los deseos e intereses del propio agente, es decir, son razones egocéntricas. Sin embargo, aceptar casos donde existe deliberación e incluso comunicación de razones permite postular momentos de transición hacia el modo humano y lingüístico de operar con razones. Hay razones sin lenguaje pero también hay razones que solo el lenguaje permite tener, cuya naturaleza es eminentemente lingüística o necesita del lenguaje para expresarse, como las razones que llevaron a Sócrates a tomar la cicuta, por poner un ejemplo. Pero también es preciso suponer una infraestructura racional no lingüística que permita ya actuar a la luz de razones y, al menos en algunos casos, expresarlas comunicativamente.

\section{Consideraciones finales}

Mi tesis general en este trabajo es que los animales carentes de lenguaje pueden operar en base a razones. Ante todo, indiqué que la conducta individual flexible ofrece en muchos casos una prueba suficiente de racionalidad instrumental individual. A diferencia de la acción explicable, por ejemplo, en términos de asociaciones entre estímulos, el agente individual comprende aquí las razones por las que actúa. En ausencia de lenguaje, esta racionalidad instrumental se desarrolla ante todo en el marco de problemas prácticos como el del uso y la fabricación de herramientas.

En este punto se pusieron en evidencia algunos problemas filosóficos referidos a la naturaleza de las razones y de los procesos de pensamiento 
involucrados en la racionalidad instrumental. Me incliné por explicar las razones como representaciones mentales en el marco de una relación intencional. Esto requiere para algunos autores una capacidad de auto-cognición, que fue preciso reconocer. Respecto de los procesos de razonamiento, para algunos autores estos serian no solo meta-cognitivos sino también inferenciales, lo cual requeriria lenguaje (contra mi tesis general). Para deflacionar estas exigencias me pareció adecuado pensar la deliberación como un proceso meta-cognitivo que opera sobre series de representaciones asociadas.

Finalmente, consideré que se podía extender la tesis general hasta la capacidad para comunicar razones sin lenguaje. En base a experimentos con chimpancés que, según Tomasello, poseen una infraestructura comunicativa, sostuve que algunas conductas individuales pueden interpretarse como dar razones comunicativamente.

Este recorrido me ha llevado a las siguientes conclusiones. Primero, resulta cuestionable restringir la capacidad de deliberación a los agentes lingüísticos, tanto por la fuerza de los contraejemplos como por el hecho de que existen explicaciones alternativas disponibles. Segundo, los problemas filosóficos no son fácilmente solucionables. En especial, resulta dificil ofrecer una explicación -aunque intenté hacerlo- de cómo son los procesos deliberativos que, una vez reconstruidos lingüísticamente, tienen la forma de una inferencia. Esto mantiene la tensión entre la evidencia disponible -y creciente- referida a la acción animal compleja y los recelos filosóficos sobre el pensamiento sin lenguaje.

\section{Bibliografia}

Andrews, K., Do Apes Read Minds. Toward a New Folk Psychology, Cambridge Mass./ Londres: The MIT Press, 2012.

Bermúdez, J.L., Thinking without Words, Oxford: Oxford University Press, 2003. http:// dx.doi.org/10.1093/acprof:oso/9780195159691.001.0001

Boesch, C. y H. Boesch, "Tool use and tool making in wild chimpanzees", en: Folia Primatologica, v. LIV (1990), pp. 86-99. http://dx.doi.org/10.1159/000156428

Camp, E., "Thinking with Maps", en: Philosophical Perspectives, v. XXI, 1 (2007), pp. 145-182. http://dx.doi.org/10.1111/j.1520-8583.2007.00124.x

Crelier, A., "Distancia y mundo. La diferencia específica de los animales humanos sobre el trasfondo de la tradición filosófica", en: Nuevas Fronteras de Filosofia Práctica, v. I (2013), pp. 84-105. Disponible en: http:/ / nuevasfronterasdefilosofiapractica. com/2013/09/23/numero-1-agosto-de-2013/

Davidson, D., "Tener la intención”, en: Ensayos sobre acciones y sucesos, Barcelona: Crítica, 1995

ARETÉ Revista de Filosofia, vol. XXVIII, N² 2, 2016 / ISSN 1016-913X 
Dickinson, A. y B.W., Balleine, "Causal Cognition and Goal-Directed Action", en: Heyes, C. y L. Huber, The Evolution of Cognition, Cambridge Mass.: Cambridge University Press, 2000.

Glock, H.-J., “Can Animals Act for Reasons?”, en: Inquiry, v. LII, 3 (2009), pp. 232-254. http://dx.doi.org/10.1080/00201740902917127

Glock, H.-J., La mente de los animales. Problemas conceptuales, Oviedo: KRK Ediciones, 2009.

Hacker, P.M.S., Human Nature: the Categorial Framework, Blackwell Publishing, 2007. http://dx.doi.org/10.1002/9780470692165

Hurley, S., "Making sense of animals", en: Hurley, S. y M. Nudds (eds.), Rational animals?, Oxford: Oxford University Press, 2006. http://dx.doi.org/10.1093/acpro f:oso/9780198528272.003.0006

Kant, I., Crítica de la razón pura, Buenos Aires: Colihue, 2009.

Kenny, A., Aquinas and Mind, Londres: Routledge, 1993.

Malcolm, N., "Thoughtless Brutes", en: Proceedings and Addresses of the American Philosophical Association, v. XLVI (1972-1973), pp. 5-20.

McDowell, J., Mind and World, Cambridge Mass.: Harvard University Press, 1996.

McIntyre, A., Animales racionales y dependientes, Barcelona: Paidós, 2001.

Millikan, R.G., "Pushmi-Pullyu representations", en: Tomberlin, J. (ed.), Philosophical Perspectives, Atascadero: Ridgeview Publishing, 1996, pp. 185-200.

Papineau, D., "The Evolution of Means-End Reasoning", en: Royal Institute of Philosophy Supplement, v. XLIX (2001), pp. 145-178. http://dx.doi.org/10.1017/ S1358246100007153

Tomasello, M., Origins of Human Communication, Cambridge Mass.: The MIT Press, 2008.

Recibido: 14/11/14

Aceptado: 24/09/15 